\title{
Verification of causal influences of reasoning skills and epistemology on physics conceptual learning
}

\author{
Lin Ding \\ Department of Teaching and Learning, The Ohio State University, Columbus, Ohio 43210, USA
}

(Received 22 March 2014; published 14 July 2014)

\begin{abstract}
This study seeks to test the causal influences of reasoning skills and epistemologies on student conceptual learning in physics. A causal model, integrating multiple variables that were investigated separately in the prior literature, is proposed and tested through path analysis. These variables include student preinstructional reasoning skills measured by the Classroom Test of Scientific Reasoning, pre- and postepistemological views measured by the Colorado Learning Attitudes about Science Survey, and preand postperformance on Newtonian concepts measured by the Force Concept Inventory. Students from a traditionally taught calculus-based introductory mechanics course at a research university participated in the study. Results largely support the postulated causal model and reveal strong influences of reasoning skills and preinstructional epistemology on student conceptual learning gains. Interestingly enough, postinstructional epistemology does not appear to have a significant influence on student learning gains. Moreover, pre- and postinstructional epistemology, although barely different from each other on average, have little causal connection between them.
\end{abstract}

DOI: $10.1103 /$ PhysRevSTPER.10.023101

PACS numbers: 01.40.Fk, 01.40.Ha, 01.40.gf

\section{INTRODUCTION}

One of the fundamental tasks in education research is to understand what influences student learning [1]. Evidently, this task is important because it can directly shape the ways we approach teaching and learning. To put it more specifically, it is only when we have a clearer picture of what influences learning that we can develop and implement effective instructional techniques [1]. Physics education research, as a pioneer in this regard, has put forward several key factors that are postulated to exert significant influences on student learning. Among them are student reasoning skills [2-5] and epistemological sophistication levels $[6,7]$ - two factors that have recently become the focus of many studies.

A host of literature suggests that students' reasoning skills and epistemological views about knowledge and learning may be important predictors of their conceptual gains in physics [2-7]. Empirical studies on this matter largely rely on either correlation analysis or small-scale case studies to hint at possible causal relationships between these variables. While these research approaches are undoubtedly useful for initial exploration, further investigations are needed to directly verify (or falsify) the previously hypothesized causality in an integrative manner.

In light of this need, the current study uses path analysis (see Methodology) to concurrently examine the causal

Published by the American Physical Society under the terms of the Creative Commons Attribution 3.0 License. Further distribution of this work must maintain attribution to the author(s) and the published article's title, journal citation, and DOI. influences of reasoning skills and epistemological sophistication on conceptual learning in college-level introductory mechanics. Results of the study speak directly to the previously proposed causality and provide some evidence for the generalizability of prior findings. In what follows, we first introduce a theoretical background of the study, followed by descriptions of research methods. Key findings from path analysis are reported, and important implications for introductory-level physics instruction are discussed.

\section{THEORETICAL BACKGROUND}

Drawing on prior relevant studies, a causal model is depicted in Fig. 1 to capture a set of reasonable and parsimonious relations among reasoning skills, epistemological sophistication, and conceptual learning gains. Here, the arrows represent potential causal influences; the direction of each arrow indicates the flow of the hypothesized influence from causal variables to effect variables.

In Fig. 1, reasoning is depicted to have a direct influence on student conceptual learning (arrow I). A body of literature has proposed this causal relationship. According to

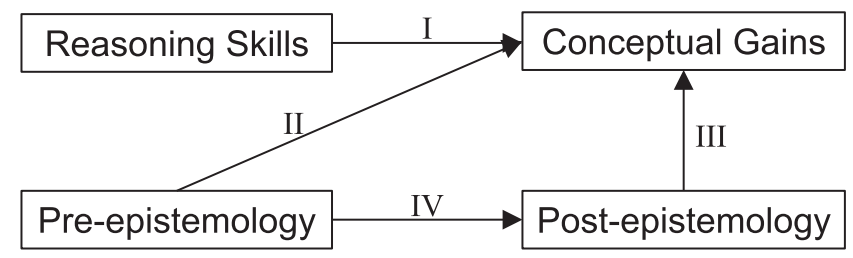

FIG. 1. A causal model of reasoning and epistemology in relation to conceptual learning. Arrows represent influence flow from cause to effect. 
Lawson [8], reasoning can be generally defined as utilizing mental strategies, plans, and rules to devise explanations for phenomena of direct observation. He conducted a series of studies, consistently postulating and demonstrating that formal reasoners, due to their higher level of reasoning and capability of abstract thinking, were more successful in learning science concepts than concrete or transitional reasoners (those at lower levels of reasoning and only capable of thinking through concrete objects or deficient of abstract thinking) [8]. In physics, Meltzer framed conceptual learning as a causal function of some "hidden variables," such as reasoning skills [9]. He further argued that math skills could also be influenced by reasoning skills, which in turn would result in a spurious correlation between student performance on physics concept tests and on math tests. Recently, Coletta, Phillips, and Steinert [3] discovered that conceptual gains in physics could vary significantly from one student group to another, relating ceteris paribus to their reasoning skills. This result notwithstanding, the correlation between student preinstructional reasoning skills (measured by the Classroom Test of Scientific Reasoning [10]) and conceptual learning gains (measured by the Force Concept Inventory [11]) seemed to be fairly robust. In fact, numerous studies have reported significant correlations in the range of $r=0.27$ and $r=0.51$ [2-5,12,13]. Given these findings, Coletta and colleagues hypothesized that preinstructional reasoning skills played a causal role in the observed variations in student conceptual learning gains [2,3].

Student epistemological views are also considered as a causal agent of conceptual gains (arrows II and III in Fig. 1). Prior studies, both theoretical and empirical, have repeatedly pointed to this relationship. In a general sense, epistemology is referred to as ideas about the nature of knowledge and the nature of learning [6,7]. Given its context dependent property, a learner's epistemology is often deemed to situate at the nexus of their beliefs, attitudes, and expectations [7]. Lising and Elby [6] conducted a case study, periodically interviewing a student taking an introductory physics course throughout an entire school year. After observing and analyzing the student's approaches to physics problems, the researchers concluded that much of the student's difficulty in learning physics in fact stemmed from her epistemic failure in connecting everyday sense making and formal thinking in physics. From this conclusion, Lising and Elby [6] further argued that a student's epistemological stance could "have a direct, causal influence on learning of physics." This argument seems consistent with many correlation results from largerscale survey studies. Sahin [14], for example, used the Maryland Physics Expectations Survey [15] to gauge students' epistemologies in a problem-based physics course and found that students' responses significantly correlated to their course grades. In a similar vein, Perkins and colleagues [16] measured students' epistemological views before and after instruction using the Colorado Learning Attitudes about Science Survey [17]. Both the pre and post results correlated with student gains on physics concept tests, signifying a possible causal connection between epistemology and conceptual learning in physics. Similar findings have been frequently reported across student populations from different nations [14,18].

Another recurrent pattern regarding student epistemologies is that overall they seem unlikely to improve through formal instruction [17]. Research has shown that students continued to maintain novicelike views about physics and learning physics even after taking introductory courses [17]. In other words, there appears to be little effect of formal instruction on student epistemological growth, and what students already hold in their views prior to instruction seems to persist afterwards. In this sense, previous studies suggest a possible influence from preinstructional to postinstructional epistemology (arrow IV in Fig. 1).

Piecing all the above into one system, Fig. 1 sketches out a basic causal model anchored in prior relevant research. The goal of the study is to test this model with an attempt to concurrently verify (or falsify) these causal links.

\section{METHODS}

Participants. This study was conducted with 167 Chinese first-year college students taking a calculus-based introductory mechanics course at a research university in East China. This is a comprehensive higher institution, ranking among the top 50 nationally and equivalent to large state research universities in the United States. All students were science or engineering majors, taking physics as a mandatory course to fulfill degree requirements. In this study, the introductory mechanics course was traditionally taught by a senior physics instructor in a large lecture hall. The course content followed canonical textbook topics, primarily including kinematics, Newton's laws, linear momentum, circular motion, energy, angular momentum, and rotational dynamics. Students attended three hour-long lectures and a one-hour recitation every week, similar in format to those traditional physics courses taught in the United States. In addition, students participated in a 2-hour lab each week, conducting mostly cookbooklike lab activities.

Instruments. We administered three published instruments to measure, respectively, student reasoning skills, epistemological views, and learning gains on Newtonian concepts. These instruments were Classroom Test of Scientific Reasoning (CTSR) [10], Colorado Learning Attitudes about Science Survey (CLASS) [17], and Force Concept Inventory (FCI) [11]. All the tests are research based and have been broadly used for empirical investigations and instructional practices. The Mandarin version of the tests (the same as those used in previously reported work $[19,20])$ was adopted for the current study. All the tests went through validity checks using interviews 
with a dozen Chinese students from a similar population to ensure the translation did not cause misinterpretation. We also calculated the reliability indices of the tests, which ranged from 0.74 to 0.81 , suggesting a consistency of measurements adequate for subsequent analysis.

The preinstructional CTSR and FCI were conducted in the first week of the mechanics course as in-class tests. Students spent a maximum of 30 min completing each test. The pre CLASS was administered in the first lab within $15 \mathrm{~min}$. On the last day of class, post FCI and post CLASS took place. As before, students were allowed 30 and 15 min, respectively to finish each test. In all cases, the tests were paper based; students were encouraged to take them seriously and received a small amount of course credit for participation.

Path analysis. Simply put, path analysis is a multiregression technique designed to test cause-effect relationships [21]. A unique strength of path analysis is that it can handle directed complex dependencies among multiple variables. In other words, besides revealing the existence of possible relations (like correlations), path analysis can test their directionality. While basic regression may work well for simple relations, they often fall short of the capability to deal with complex relations that are hierarchical; for example, a chain relation in which variable $A$ influences variable $B$, which in turn influences variable $C$ (i.e., $A \rightarrow B \rightarrow C$ ). In this case, path analysis is a suitable approach. Moreover, path analysis provides a set of fit statistics to evaluate the robustness of proposed causal relations, thereby empirically testing the likelihood of the correctness of a model [21].

Strictly speaking, causality can never be proved but can only be inferred to a reasonably acceptable level [21]. From the philosophy of science standpoint, causality research in essence represents efforts of falsification. In this sense, the current study (albeit aiming for verifying a causal model) can in fact be viewed as an attempt to falsify the proposed causal links. While a positive outcome does not definitively "prove" causality, it does show that the model has sustained our attempt of falsification and therefore its likelihood of being true is increased [21].

\section{RESULTS}

Based on the data collected from the 167 participants, we recorded for each student a set of five scores, representing, respectively, their performance on CTSR, CLASS (pre and post), and FCI (pre and post). Conceptual learning changes were calculated from the normalized FCI gains. Table I shows the overall averages (percentage).
As seen, student reasoning skills measured by the CTSR were roughly at the so-called transitional stage (between concrete and formal levels), which is typical of many college freshmen in previous studies [19]. Student epistemological sophistication levels (both before and after instruction) measured by the CLASS were also comparable to those in the prior literature [17]. Not surprisingly, there was little change in the CLASS results after students took the mechanics course. Conversely, the increase on the FCI was notable, resulting in an average normalized gain of decent size.

We first checked the normality of the data prior to path analysis. According to Kline [22], an absolute value of skewness greater than 2 and absolute kurtosis greater than 7 are considered as extreme and may indicate a violation of normal distribution. In the current study, all score distributions showed absolute skewness less than 1.7 and absolute kurtosis below 5.5, suggesting no severe deviation from normal distribution.

Before interpreting the path analysis results, it is crucial to examine the fit of the model. Prior literature has established a set of standards for judging the robustness of a model under path analysis, including the overall chi-square test $\left(\chi^{2}\right)$, root-mean-square residuals (RMSR), root-mean-square error of approximation (RMSEA), goodness-of-fit index (GFI), adjusted goodness-of-fit index (AGFI), comparative fit index (CFI), and normed fit index (NFI) [21]. These standards (although admittedly daunting) are created to help researchers determine the extent to which a model is acceptable. Ideally, a viable model should give rise to an insignificant $\chi^{2}(p>0.05)$, small RMSR and RMSEA $(<0.050)$, and high fit indices $(\geq 0.90)$ [21]. In our study, the model yielded satisfactory values: $\chi^{2}=0.01$ $(p=0.92), \quad \mathrm{RMSR}=0.003, \quad \mathrm{RMSEA}=0.000, \quad \mathrm{GFI}=$ 1.00, AGFI $=0.99, \mathrm{CFI}=1.00, \mathrm{NFI}=0.99$, suggesting a good fit between the data and the hypothesized model. (To avoid digression, details of fit indices are omitted. See Ref. [21] for more information.)

Figure 2 shows the resultant model with standardized path coefficients. These coefficients represent the size of the influence from causal variables to effect variables. Typically, path values in the range of 0.05 and 0.10 are considered as "small" influences, values ranging from 0.10 to 0.25 are "moderate," and values above 0.25 are "large" $[21,22]$. In our model, student reasoning skills have a large, direct influence (0.31) on conceptual learning gains, and similarly preinstructional epistemology appears to have a moderate, direct influence (0.18) on student conceptual gains. To further check the significance of these paths, a $t$

TABLE I. Average test scores (\%) and FCI normalized gain (average of individual students' normalized gains).

\begin{tabular}{lcccccc}
\hline \hline Tests & CTSR & Pre CLASS & Post CLASS & Pre FCI & Post FCI & FCI Norm Gain \\
\hline Avg \pm SD & $75.2 \pm 15.8$ & $57.5 \pm 17.0$ & $58.4 \pm 16.0$ & $60.6 \pm 8.4$ & $81.8 \pm 6.4$ & $52.1 \pm 18.9$ \\
\hline \hline
\end{tabular}




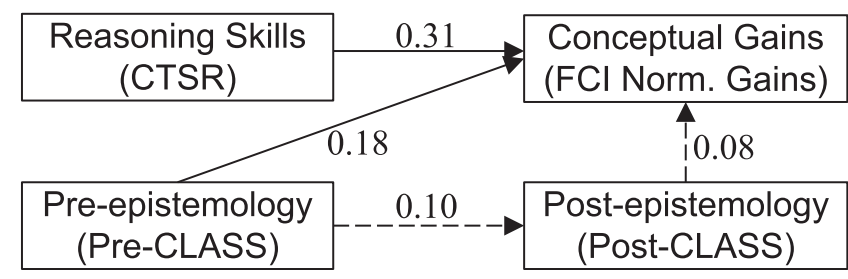

FIG. 2. A resultant model with standardized path coefficients. Dashed arrows indicate insignificant causal paths.

test of the coefficients is conducted and results show that both paths are not due to random coincidence (reasoning: $p<0.001$; preepistemology: $p=0.019$ ).

On the other hand, student postinstructional epistemology has a direct but small (0.08) influence on conceptual learning gains. A $t$ test confirms that this path is indeed insignificant $(p=0.29)$. Moreover, student preepistemology has a small-to-moderate influence on postepistemology $(0.10)$. As with the previous case, this path is shown to be insignificant $(p=0.21)$ and hence carries little weight in the model.

\section{DISCUSSION}

By and large our model, derived from prior theories and empirical investigations, is supported by path analysis. It is clear from the results that two factors have noticeable influences on student conceptual learning measured by the FCI. One is scientific reasoning skills that students have before coming to class, and the other is student preinstructional views about the nature of physics and learning physics. These two factors represent two important but different domains of learning sciences: logical thinking and epistemological stance. The former, in a broader context, is linked to cognitive and inquiry skills that may span beyond science content. For example, Coletta and Phillips [3] used a general test on verbal and math reasoning (Scholastic Aptitude Test) to differentiate students and found the results correlated to FCI gains equally well as CTSR scores. It is possible that more general skills than those measured by the CTSR may play a role in learning physics. That said, our findings confirmed the causal influence of scientific reasoning on student gains in Newtonian concepts. As for the other factor of epistemology, it touches upon learners' philosophical stance. It is broadly believed that students approach learning differently based on how they view knowledge. Those who view physics as a hierarchically structured, coherent enterprise are more likely to achieve learning through meaningful knowledge construction and sense making than those who view it as a body of disconnected facts. Our results supported this notion and provided evidence to illustrate a "direct, causal influence" of student preinstructional epistemology on conceptual learning in mechanics.

Interestingly enough, student postepistemology in our model appeared to have little effect on conceptual gains.
One possible account is that the FCI gains represent a past event (a pre-to-post shift), whereas postepistemology is a "state of mind" after the change in FCI has occurred. Certainly, later occurring events cannot (from a logical standpoint) impose causal influences on earlier events. Another explanation is that although students' views measured by the CLASS on average changed little from pre- to postinstruction, substantial changes at the individual students' level may still exist. However, these individual changes may not represent any meaningful pattern, thereby attenuating the causal connection between epistemology and conceptual learning that existed in the preinstructional case. In other words, the causal influence of epistemology on conceptual learning prior to instruction diminished after the mechanics course, perhaps because the physics instruction in our study only produced unsystematic changes among the individual students. Indeed, the pre- and postepistemologies measured by the CLASS in our study yielded a close-to-zero correlation, suggesting a lack of regularity in the changes at the individual students' level. This may also explain why there is little influence from preepistemology to postepistemology in our case. It is worth noting that the study was conducted in a traditionally taught physics course and thus represented a typical case in which the instructor made no deliberate effort to explicate or promote students' epistemological development in teaching. Had more instructional attention been directed to this important aspect, the resultant student epistemology and its influence on conceptual learning might have been more encouraging than what was seen here.

The implications of the study are twofold. From the research perspective, our findings provide confirmatory as well as falsifying evidence to the proposed causality regarding reasoning, epistemology, and physics learning. It is worth noting that the model tested herein synthesizes multiple variables (that were previously investigated separately) into one system and aims directly at their causal relationships. This model, although shown to be mostly decent, by no means represents a thorough account of all causal relations. For instance, learning behaviors may mediate the influence of epistemology on conceptual learning as suggested by Kortemeyer [23]. Therefore, adding more variables into the current model may further increase its veridicality. To this end, the model we investigated only represents a parsimonious case whose generalizability is tested in the current study.

From the pedagogical viewpoint, our findings highlight the importance of some often overlooked learning aspects other than those that are conventionally emphasized in instruction (such as acquisition of content knowledge). Since thinking skills and epistemological understandings can have direct, causal influences on learning outcomes, it behooves researchers and educators to include cultivation of student reasoning and epistemological growth as part of their instructional goals. Certainly, pedagogical practices 
must follow in order to successfully fulfill these goals. Fortunately, recent efforts have been increasingly directed towards this topic and new development is starting to emerge. Nevertheless, much work still remains. After all, physics education is no easy task and it involves far more factors than what can be captured in one study.

\section{ACKNOWLEDGMENTS}

The author wishes to thank Katherine Mollohan for many insightful discussions. This work is partially supported by the National Science Foundation (NSF Grant No. DRL 1252399).
[1] J. D. Bransford, A. L. Brown, and R. R. Cocking, How People Learn: Brain, Mind, Experience, and School, (Natl. Acad. Press, Washington, DC, 2000), Expanded ed.

[2] V. P. Coletta and J. A. Phillips, Interpreting FCI scores: Normalized gain, preinstruction scores, and scientific reasoning ability, Am. J. Phys. 73, 1172 (2005).

[3] V. P. Coletta, J. A. Phillips, and J. J. Steinert, Interpreting Force Concept Inventory scores: Normalized gain and SAT scores, Phys. Rev. ST Phys. Educ. Res. 3, 010106 (2007).

[4] P. Nieminen, A. Savinainen, and J. Viiri, Relations between representational consistency, conceptual understanding of the force concept, and scientific reasoning, Phys. Rev. ST Phys. Educ. Res. 8, 010123 (2012).

[5] J. C. Moore and L. J. Rubbo, Scientific reasoning abilities of nonscience majors in physics-based courses, Phys. Rev. ST Phys. Educ. Res. 8, 010106 (2012).

[6] L. Lising and A. Elby, The impact of epistemology on learning: A case study from introductory physics, Am. J. Phys. 73, 372 (2005).

[7] D. Hammer and A. Elby, Tapping epistemological resources for learning physics, J. Learn. Sci., 12, 53 (2003).

[8] A. E. Lawson, The nature and development of scientific reasoning: A synthetic view, Int. J. Sci. Math. Educ. 2, 307 (2004).

[9] D. E. Meltzer, The relationship between mathematics preparation and conceptual learning gains in physics: A possible "hidden variable" in diagnostic pretest scores, Am. J. Phys. 70, 1259 (2002).

[10] A.E. Lawson, The development and validation of a classroom test of formal reasoning, J. Res. Sci. Teach. 15, 11 (1978).

[11] D. Hestenes, M. Wells, and G. Swackhamer, Force Concept Inventory, Phys. Teach., 30, 141 (1992).

[12] K. Diff and N. Tache, From FCI to CSEM to Lawson test: A report on data collected at a community college, in Proceedings of the 2007 Physics Education Research Conference, edited by L. Hsu, C. Henderson, and L. McCullough (AIP, New York, 2007), Vol. 951, pp. 85-87.

[13] M. Semark, R. Dietz, R. Pearson, and C. Willis, Predicting FCI gain with a nonverbal intelligence test, in Proceedings of the 2012 Physics Education Research Conference, edited by P. Engelhardt, A. Churukian, and N. S. Rebello (AIP, New York, 2012), Vol. 1513, pp. 378-381.

[14] M. Sahin, Correlations of students' grades, expectations, epistemological beliefs and demographics in a problembased introductory physics course, Int. J. Environ. Sci. Educ. 4, 169 (2009).

[15] E. F. Redish, J. M. Saul, and R. N. Steinberg, Student expectations in introductory physics, Am. J. Phys. 66, 212 (1998).

[16] K. Perkins, W. Adams, S. Pollock, N. Finkelstein, and C. Wieman, Correlating student beliefs with student learning using the Colorado Learning Attitudes about Science Survey, in Proceedings of the 2004 Physics Education Research Conference, edited by J. Marx, P. Heron, and S. Franklin (AIP, New York, 2005) Vol. 790, pp. 61-64.

[17] W. K. Adams, K. K. Perkins, N. S. Podolefsky, M. Dubson, N. D. Finkelstein, and C. E. Wieman, New instrument for measuring student beliefs about physics and learning physics: The Colorado Learning Attitudes about Science Survey, Phys. Rev. ST Phys. Educ. Res. 2, 010101 (2006).

[18] S. Sharma, P. K. Ahluwalia, and S. K. Sharma, Students' epistemological beliefs, expectations, and learning physics: An international comparison, Phys. Rev. ST Phys. Educ. Res. 9, 010117 (2013).

[19] L. Ding, Detecting progression of scientific reasoning among university science, and engineering students, in Proceedings of the 2013 Physics Education Research Conference, edited by P. V. Engelhardt, A. D. Churukian, and D. L. Jones (Am. Assoc. Physics Teachers, College Park, MD, 2014), pp. 125-128; http://dx.doi.org/2200/ 20140224185550632T.

[20] P. Zhang and L. Ding, Large-scale survey of Chinese precollege students' epistemological beliefs about physics: A progression or a regression?, Phys. Rev. ST Phys. Educ. Res. 9, 010110 (2013).

[21] N. Blunch, Introduction to Structural Equation Modeling (Sage, Thousand Oaks, CA, 2008).

[22] R. Kline, Principles and Practice of Structural Equation Modeling (Guilford Press, New York, 2011).

[23] G. Kortemeyer, Correlations between student discussion behavior, attitudes, and learning, Phys. Rev. ST Phys. Educ. Res. 3, 010101 (2007). 